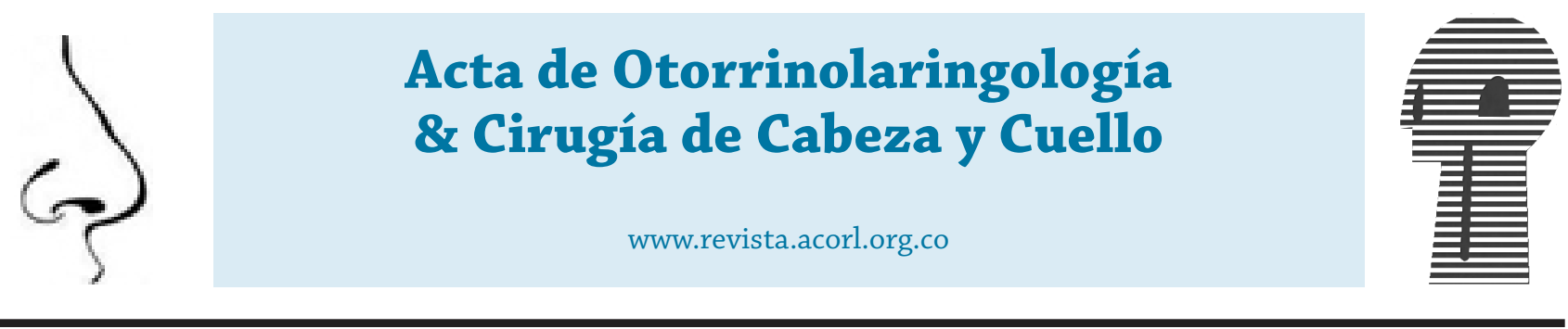

Trabajos originales

\title{
Complicaciones posquirúrgicas del manejo del dorso nasal con la técnica cartílago en puente
}

\section{Post-surgical complications of the management of the nasal dorsum with the cartilage-bridging technique}

\author{
Clara Sofía Oñate-Carrillo*, Juliana Montero-Cortés**, Andrea Hortua-Bayona***, \\ Armando Rojas-Salazar ${ }^{* * * *}$ \\ * Especialista en Otorrinolaringología, Facultad de Medicina, Universidad de Cartagena. Cartagena, Colombia \\ ** Especialista en Otorrinolaringología, Clínica del Bosque. Cartagena, Colombia. \\ *** Especialista en Otorrinolaringología, Unidad de Otorrinolaringología en Bucaramanga. Bucaramanga, Colombia. \\ ${ }^{* * * *}$ Especialista en Otorrinolaringología; docente del programa de Otorrinolaringología, Facultad de Medicina. Universidad de \\ Cartagena. Cartagena, Colombia.
}

Forma de citar: Oñate-Carrillo CS, Montero-Cortés J, Hortua-Bayona A, Armando Rojas-Salazar A. Complicaciones posquirúrgicas del manejo del dorso nasal con la técnica cartílago en puente. Acta otorrinolaringol. cir. cabeza cuello. 2021;49(1):36-42. DOI. 10.37076/acorl.v49i1.540

\section{INFORMACIÓN DEL ARTÍCULO}

Historia del artículo:

Recibido: 9 de julio de 2020

Evaluado: 28 de febrero de 2021

Aceptado: 22 de marzo de 2021

Palabras clave (DeCS):

Rinoplastia, procedimientos quirúrgicos nasales, cartílagos nasales, complicaciones posoperatorias.

\section{RESUMEN}

Introducción: en Cartagena, desde hace aproximadamente una década, se está realizando una nueva técnica de injerto dorsal denominada cartílago en puente (CEP), realizada en rinoplastias cerradas por un solo otorrinolaringólogo. No obstante, no existen estudios que caractericen las complicaciones de esta técnica. Objetivo del estudio: caracterizar las complicaciones posquirúrgicas del manejo del dorso nasal con CEP en pacientes sometidos a una rinoplastia. Metodología: estudio observacional descriptivo, de corte transversal y de carácter retrospectivo, que abarca los registros clínicos de pacientes sometidos a una rinoplastia cerrada con la técnica CEP entre 2013 y 2016. Se midieron variables sociodemográficas, características del procedimiento y se registraron las complicaciones detectadas durante el seguimiento posoperatorio: aparición de infección, extrusión, desplazamiento y reabsorción del injerto Resultados: se identificaron 882 historias clínicas. La mediana de edad fue de 28 años, siendo el $81 \%$ del género femenino. La rinoplastia fue primaria en 80,6 $\%$, el material del injerto dorsal fue solo cartílago en $80,6 \%$, Gore-Tex $®$ en $1,7 \%$ y una combinación de cartílago y Gore-Tex ${ }^{\circledR}$ en $17,7 \%$. La mediana de duración 
de las rinoplastias fue de 78 minutos (rango intercuartílico [RIC]: 72-85), siendo el principal procedimiento adicional la septoplastia en $82,2 \%$. El 18,2 \% de los pacientes presentaron complicaciones. El desplazamiento fue la principal complicación reportada $(14,7 \%)$, seguida de la reabsorción $(4,0 \%)$ e infección $(0,1 \%)$. Conclusiones: la técnica CEP mostró como principales complicaciones el desplazamiento y la reabsorción del injerto, con una frecuencia similar a la descrita en otros estudios.

\section{ABSTRACT}

Key words (MeSH):

Nasal Surgical Procedures; Nasal Cartilages; Postoperative Complications.
Introduction: In Cartagena, a new dorsal graft technique called Cartilage in Bridge (CEP) has been carried out for approximately a decade, performed in rhinoplasties closed by a single otorhinolaryngologist. However, there are no studies that characterize the complications of this technique. Objective: To characterize postoperative complications of the management of the nasal dorsum with bridge cartilage in patients undergoing rhinoplasty. Methodology: Retrospective, descriptive, observational cross-sectional study, covering clinical records of patients who underwent closed rhinoplasty with the bridge cartilage technique between 2013 and 2016. Sociodemographic variables, characteristics of the procedure, and complications detected during follow-up were recorded postoperative: appearance of infection, extrusion, displacement, and reabsorption of the graft Results: 882 medical records were identified. The median age was 28 years, being $81 \%$ female. Rhinoplasty was primary in $80.6 \%$, the dorsal graft material was only cartilage in $80.6 \%$, Gore-Tex ${ }^{\circledR}$ in 1.7 $\%$ and combination of cartilage and Gore-Tex ${ }^{\circledR}$ in $17.7 \%$. The median duration of the rhinoplasties was 78 minutes (ICR: 72-85), with the main additional procedure being septoplasty in $82.2 \% .18 .2 \%$ of the patients presented complications. Displacement $(14.7 \%)$ was the main complication reported, followed by reabsorption $(4.0$ $\%$ ) and infection $(0.1 \%)$. Conclusions: The cartilage bridge technique showed graft displacement and reabsorption as main complications, with a frequency similar to that described in other studies.

\section{Introducción}

La rinoplastia es uno de los procedimientos estéticos más complejos realizados por cirujanos plásticos y otorrinolaringólogos en todo el mundo, lo que la convierte en una de las cirugías estéticas más realizadas junto con la mamoplastia de aumento y la liposucción (1). La dificultad de este procedimiento se le atribuye a la complejidad del área anatómica, que incluye cartílago, estructuras óseas y una cubierta de piel suave y delgada (2); además, las distintas técnicas quirúrgicas existentes representan un reto adicional debido a su complejidad técnica.

En la rinoplastia abierta se realiza una incisión columelar que permite la visión directa del campo operatorio, lo que permite la identificación de defectos; sus desventajas principales son las cicatrices y los hematomas. En la técnica de rinoplastia cerrada el abordaje incisional se realiza con una transfixión a través de la maxila y la premaxila; esta requiere una mayor habilidad del cirujano y se puede realizar de forma ambulatoria sin necesidad de hospitalizar al paciente (3-5).

El abordaje apropiado para cada caso dependerá de la preferencia y la experticia del cirujano. En general, no hay evidencia que demuestre que un abordaje quirúrgico dará mejores resultados que otro. Para pacientes que requieran una disminución de giba dorsal y mínimos cambios en la punta de la nariz se puede realizar el abordaje cerrado; por el contrario, si son necesarias grandes correcciones en la punta nasal o injertos cartilaginosos es más conveniente un abordaje abierto (6).

Estudios demuestran que más del $50 \%$ de cirujanos con experiencia en cirugía plástica facial prefieren la rinoplastia abierta, probablemente por las comodidades mencionadas con anterioridad (7).

Dentro de los retos quirúrgicos en toda rinoplastia se encuentra el manejo del dorso nasal, que ocurre al resecar la giba osteocartilaginosa, lo que deja expuestas las estructuras anatómicas colapsadas. Para tal efecto se han desarrollado técnicas de injertos autólogos, homólogos y materiales aloplásticos que actúen de soporte, relleno y camuflaje (810). En la actualidad el injerto ideal es el cartílago autólogo debido a sus múltiples beneficios, como su maleabilidad y resistencia, además de su fácil acceso, ya que es posible obtenerlo en el mismo campo quirúrgico (11).

Sin embargo, no siempre se obtiene una cantidad abundante, por lo que se han descrito mecanismos para optimizar su uso como el machacado, troceado o en lonjas con forma de tabaco, lo cual está asociado con fascia lata o materiales 
aloplásticos como la hidroxiapatita, implantes de silicona, polietileno de alta densidad (Medpore $\AA$ ) y politetrafluoroetileno (Gore-Tex $\left.{ }^{\circledR}\right)$; estos como última opción debido a su baja biocompatibilidad (11). El uso de uno o varios materiales de injerto es decisión del cirujano tratante, así como la técnica de optimización de este.

En Cartagena, desde hace aproximadamente 10 años, se ha estado realizando el manejo del dorso nasal en rinoplastias cerradas con una técnica aún no descrita, la cual denominamos cartílago en puente (CEP). Esta consiste en tomar una lonja de cartílago o Gore-Tex ${ }^{\circledR}$ y suturar en los extremos las lonjas más cortas de cartílago apiladas, asemejando una forma de puente.

Las complicaciones del manejo del dorso nasal con cartílago machacado, troceado o en lonjas oscilan entre un 5,6 $\%$ y $16 \%$, siendo las más comunes la infección, el desplazamiento, la extrusión, la absorción y la excesiva visibilidad (8, 12-14). Estas, a su vez, dependen de factores como el tipo de técnica quirúrgica utilizada, rinoplastia (primaria o secundaria), además de procedimientos simultáneos como la septoplastia, turbinoplastia, mentoplastia, entre otros procedimientos reconstructivos y estéticos, que también influyen en la presencia de complicaciones posquirúrgicas (14). No obstante, se desconocen las características de los pacientes sometidos a una rinoplastia con el manejo del dorso con CEP, así como sus complicaciones. El objetivo de este estudio es caracterizar las complicaciones posquirúrgicas del manejo del dorso nasal con un CEP en pacientes sometidos a una rinoplastia.

\section{Metodología}

Se realizó un estudio de corte transversal de carácter retrospectivo, que abarcó todos los registros clínicos de pacientes sometidos a una rinoplastia en la Clínica Quirúrgica de Cartagena de Indias, durante el período comprendido entre 2013 y 2016. Se tuvo en cuenta como criterio de inclusión a los pacientes en los que se utilizó la técnica de manejo del dorso con CEP, cuyo seguimiento clínico estuvieran consignado en la historia clínica, se excluyeron aquellos pacientes con datos sociodemográficos y registro de complicaciones incompletos.

Se realizó un muestreo no probabilístico por conveniencia, dado que se tuvo acceso a la totalidad de la población. Inicialmente se determinaron las variables sociodemográficas: edad, género, país de residencia, estrato socioeconómico y estado civil. Luego se procedió con la caracterización de la intervención quirúrgica, y se registraron los siguientes datos: año de realización del procedimiento, tipo de rinoplastia (primaria o secundaria), material usado para el injerto dorsal (cartílago o Gore-Tex®), duración del procedimiento en minutos y realización de procedimientos quirúrgicos adicio- nales. Finalmente se registraron los datos obtenidos durante el seguimiento posoperatorio: aparición de infección (rubor, calor, dolor, secreción purulenta), extrusión (evidencia del material usado o cartílago fuera del sitio de colocación inicial), desplazamiento (cuando el injerto se encuentra alejado de su posición, pero en el mismo plano de la ubicación inicial) y reabsorción del injerto (ausencia de cartílago autólogo usado en el manejo inicial del dorso).

La base de datos recolectada se registró en Excel versión 2016 y se analizó en el paquete estadístico STATA 14. El análisis de las variables cualitativas se realizó determinando frecuencias absolutas y relativas. Para las variables continuas se utilizó la prueba de Kolmogórov-Smirnov para establecer normalidad ( $\mathrm{p} \geq 0,05$ sigue la distribución normal). De esta forma, se calcularon las medidas de tendencia central y dispersión tipo mediana (Me) y rango intercuartílico (RIC) por la naturaleza no paramétrica de estas variables, estimada por la prueba. Las complicaciones posoperatorias se evaluaron estratificando la muestra por año, tipo de rinoplastia, material usado para el injerto y realización de procedimientos adicionales. A todas ellas se les estimaron las medidas mencionadas previamente.

\section{Responsabilidades éticas}

Este estudio se realizó dentro de la normatividad de la última versión oficial de la Declaración de Helsinki. La investigación se clasifica "sin riesgo" según el artículo 11 de la Resolución 8430 de 1993 del Ministerio de Salud de la República de Colombia.

\section{Técnica quirúrgica}

La construcción del injerto dorsal de CEP requiere tres fases (Figura 1):

- Septoplastia: esta fase implica la infiltración con lidocaína más epinefrina al $2 \%$; posteriormente la incisión hemitransfixiante, la creación de túneles submucopericóndricos y la resección de cartílago cuadrangular.

- Modelación de injerto CEP: se corta una lonja de cartílago superior y fragmentos más pequeños; luego se suturan fragmentos más pequeños al borde inferior de la lonja en cada extremo con nylon 5/0 en forma de puente, de acuerdo con el defecto en dorso nasal, y se conserva el nylon para una fijación posterior.

- Fijación del CEP al septo: en esta última fase se fija la estructura al septo caudal con 1 punto de sutura. 

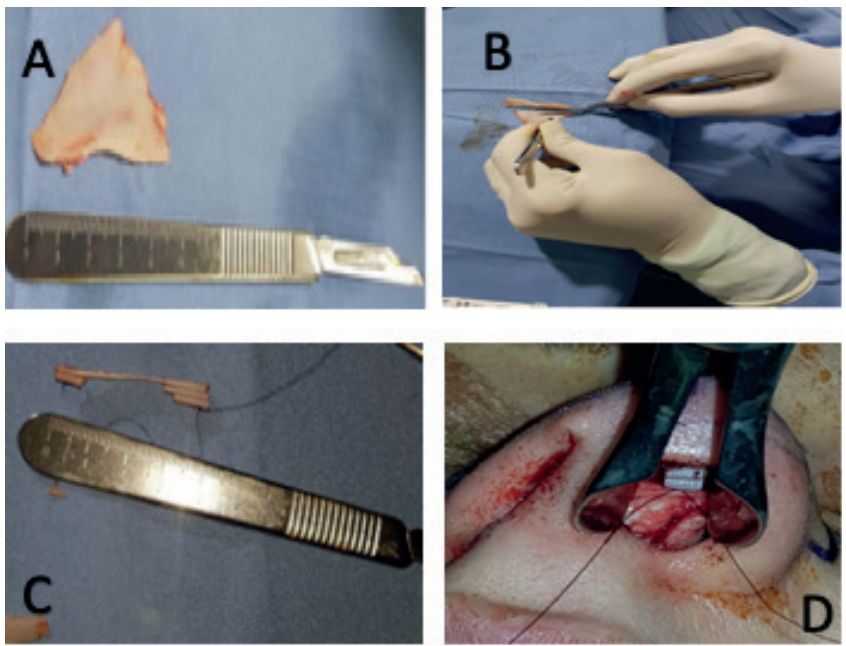

Figura 1. Fases para la construcción del injerto dorsal del CEP. A. Recolección de cartílago septal. B. Moldeamiento. C. Diseño del puente. D. Fijación del CEP al septo.

\section{Resultados}

Características generales

Se identificaron 882 historias clínicas de pacientes sometidos a una rinoplastia con manejo del dorso con injerto de CEP. La mediana de edad de los pacientes fue de 28 años (RIC: 23-34), el $81 \%$ de los procedimientos se realizaron en mujeres. Respecto a la procedencia, el 99,3\% correspondía a Colombia. El 49,1\% de los pacientes intervenidos eran estrato 3, seguido del estrato 4 con $23,6 \%$ y el 5 con $17,7 \%$. En cuanto al estado civil, el 46,5\% eran casados y el 41,2\% solteros (Tabla 1). La principal ocupación fue estudiante con un $28,2 \%$, (Figura 2).

\section{Caracterización de la intervención quirúrgica}

La distribución de los procedimientos por año fue de $23,7 \%$, $28,6 \%, 23,4 \%$ y $24,3 \%$ en $2013,2014,2015$ y 2016 , respectivamente. El 80,6 \% de las rinoplastias fueron primarias; para la construcción del injerto septal se usó solo cartílago en el $80,6 \%$ de los pacientes, solo Gore-Tex ${ }^{\circledR}$ en $1,7 \%$ y se usó una combinación de cartílago y Gore-Tex® en el 17,7 $\%$. En total, el cartílago septal se utilizó en el 98,3\% de los casos (Tabla 2).

Cabe mencionar que en todas las rinoplastias con el uso solamente de cartílago fueron las mismas clasificadas como primarias. La mediana de duración del procedimiento fue de 78 minutos (RIC: 72-85). El principal procedimiento quirúrgico adicional fue la septoplastia, con el 82,2\% y en menor proporción se encontró la realización de blefaroplastia en 3,5 $\%$, mentoplastia en 3,3\%, bichectomía en 3,2 \%, otoplastia en $1,1 \%$, liposucción de papada en 1,0\%, entre otros. Como hallazgo relevante se resalta que en todas las rinoplastias primarias se realizaron procedimientos adicionales, mientras
Tabla 1. Año de procedimiento y características sociodemográficas de la población sometida a una rinoplastia con manejo del dorso con CEP

\begin{tabular}{|c|c|c|}
\hline & $\mathbf{N}$ & $\%$ \\
\hline \multicolumn{3}{|l|}{ Año } \\
\hline 2013 & 209 & $23, .7$ \\
\hline 2014 & 252 & $28, .6$ \\
\hline 2015 & 206 & $23, .4$ \\
\hline 2016 & 215 & $24, .4$ \\
\hline Edad Me (RIC) & $28(23-34)$ & \\
\hline \multicolumn{3}{|l|}{ Sexo } \\
\hline $\mathrm{F}$ & 714 & $81, .0$ \\
\hline$M$ & 168 & $19, .0$ \\
\hline \multicolumn{3}{|l|}{ País } \\
\hline Colombia & 876 & $99, .3$ \\
\hline Estados Unidos & 3 & $0, .3$ \\
\hline Panamá & 1 & $0, .1$ \\
\hline $\begin{array}{l}\text { República } \\
\text { Ddominicana }\end{array}$ & 1 & $0, .1$ \\
\hline Venezuela & 1 & $0, .1$ \\
\hline \multicolumn{3}{|l|}{ Estrato } \\
\hline 2 & 1 & $0, .1$ \\
\hline 3 & 430 & $49, .1$ \\
\hline 4 & 207 & $23, .6$ \\
\hline 5 & 155 & $17, .7$ \\
\hline 6 & 83 & $9, .5$ \\
\hline \multicolumn{3}{|l|}{ Estado civil } \\
\hline Casado (a) & 410 & $46, .5$ \\
\hline Soltero (a) & 363 & $41, .2$ \\
\hline Unión libre & 89 & $10, .1$ \\
\hline Separado (a) & 18 & $2, .0$ \\
\hline Divorciado (a) & 2 & $0, .2$ \\
\hline
\end{tabular}

que este comportamiento se observó en solo el $14 \%$ de las secundarias.

\section{Complicaciones reportadas}

El 18,2 \% de los pacientes presentaron complicaciones. El desplazamiento fue la principal complicación reportada $(14,7 \%)$, seguida de la reabsorción $(4,0 \%)$ e infección $(0,1 \%)$. No se observó ningún caso de extrusión del injerto (Tabla 3).

Al analizar el comportamiento de las complicaciones en los años de estudio, se observó que el desplazamiento tuvo una frecuencia de $17,2 \%$ en $2013,19,0 \%$ en 2014 , y 15,5 $\%$ y $6,5 \%$ en 2015 y 2016, respectivamente. De la misma forma, la reabsorción se presentó en 2013 en 6,7 \%, con una disminución progresiva hasta llegar en 2016 al 1,9 \% (Figura 3). La distribución de las complicaciones según el tipo de rinoplastia realizada mostró una frecuencia de desplaza- 


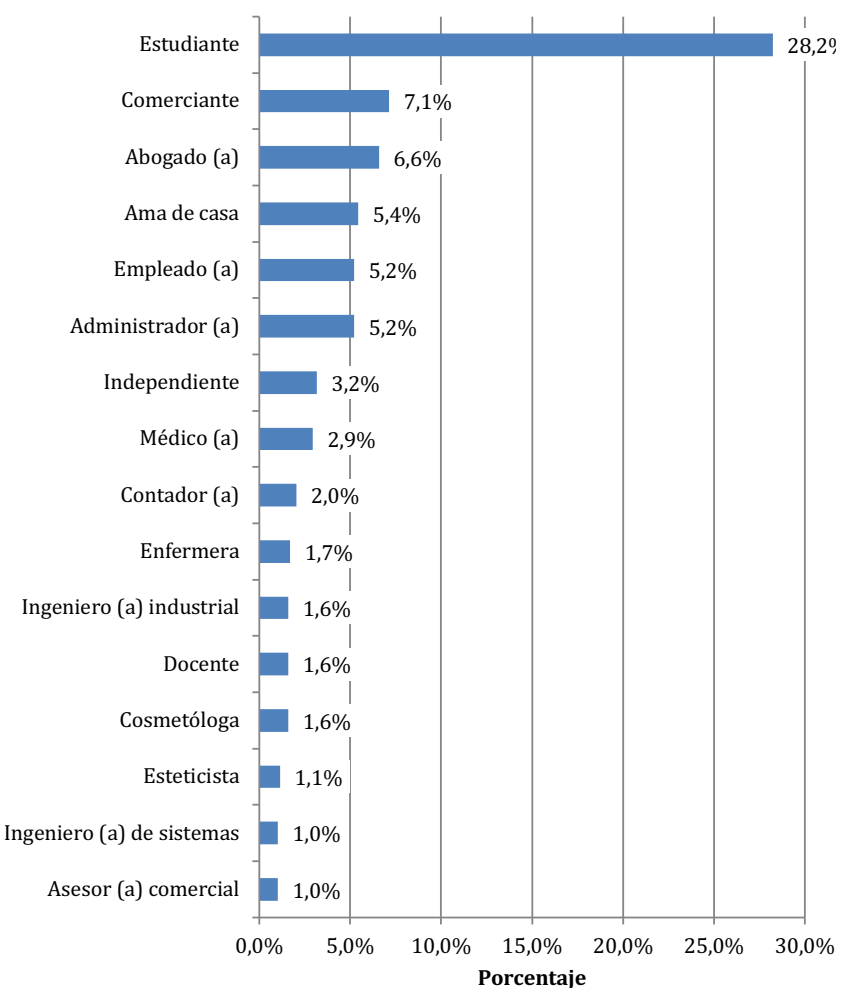

Figura 2. Principales ocupaciones de los pacientes sometidos a una rinoplastia con manejo del dorso con CEP en los cuatro años de estudio.

Tabla 2. Características del procedimiento, procedimientos adicionales y complicaciones de manejo del dorso con CEP

\begin{tabular}{|c|c|c|}
\hline & $\mathbf{N}$ & $\%$ \\
\hline \multicolumn{3}{|l|}{ Tipo de rinoplastia } \\
\hline Primaria & 711 & 80,6 \\
\hline Secundaria & 171 & 19,4 \\
\hline \multicolumn{3}{|l|}{ Material } \\
\hline Cartílago & 867 & 98,3 \\
\hline Gore-tTex $®$ & 171 & 19,4 \\
\hline Tiempo en minutos $\mathrm{Me}(\mathrm{RIC})$ & $78(72-85)$ & \\
\hline \multicolumn{3}{|l|}{ Procedimiento adicional } \\
\hline Septoplastia & 725 & 82,2 \\
\hline Blefaroplastia & 31 & 3,5 \\
\hline Mentoplastia & 29 & 3,3 \\
\hline Bichectomía & 28 & 3,2 \\
\hline Otoplastia & 11 & 13 \\
\hline Liposucción de papada & 9 & 1,0 \\
\hline Amigdalectomía & 3 & 0,3 \\
\hline Labio leporino & 2 & 0,2 \\
\hline Frontoplastia & 2 & 0,2 \\
\hline Cervicoplastia & 2 & 0,2 \\
\hline Otros & 3 & 0,3 \\
\hline
\end{tabular}

miento en las rinoplastias secundarias del $42,7 \%$ mientras que en las primarias fue de $8,0 \%$, de manera similar la reabsorción se observó respectivamente en el 13,5\% y 1,7\%.

Tabla 3. Caracterización de las complicaciones con la técnica CEP

\begin{tabular}{|l|c|c|}
\hline Complicaciones & $\begin{array}{c}\text { Frecuencia } \\
\text { absoluta }\end{array}$ & $\begin{array}{c}\text { Frecuencia } \\
\text { relativa (\%) }\end{array}$ \\
\hline Desplazamiento & 130 & $14, .7$ \\
\hline Reabsorción & 35 & $4, .0$ \\
\hline Infección & 1 & $0, .1$ \\
\hline Extrusión & 0 & $0, .0$ \\
\hline
\end{tabular}

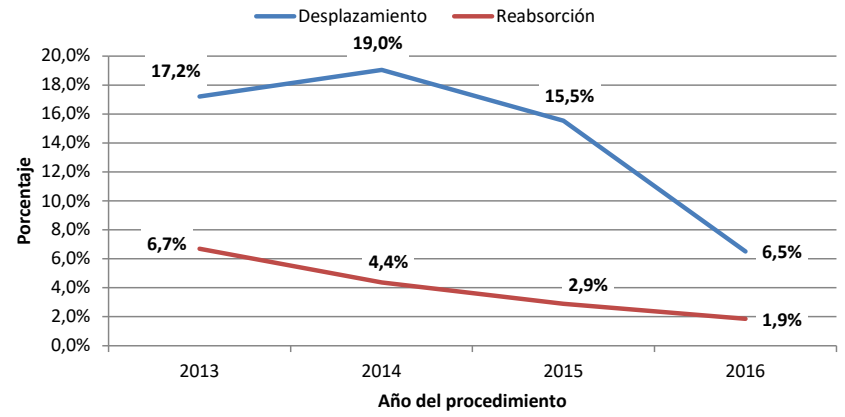

Figura 3. Comportamiento anual de las principales complicaciones en rinoplastia con el manejo del dorso con CEP.

La infección se presentó en $0,6 \%$ de las rinoplastias secundarias (Figura 4). La evaluación de las complicaciones estratificada por el tipo de material usado en el injerto mostró que en las rinoplastias con el uso solamente del cartílago hubo desplazamiento del 8,0 \% y reabsorción de $1,7 \%$; con el uso exclusivo de Gore-Tex ${ }^{\circledR}$ el desplazamiento fue de $60,0 \%$ y la reabsorción de $13,3 \%$; y con el uso de material mixto cartílago más Gore-Tex ${ }^{\circledR}$ el desplazamiento fue de $41,0 \%$, la reabsorción de $13,5 \%$ y la infección de $0,6 \%$ (Figura 5). Cabe resaltar que el único caso de infección se presentó en 2013 en una rinoplastia secundaria en la que se usaron materiales mixtos.

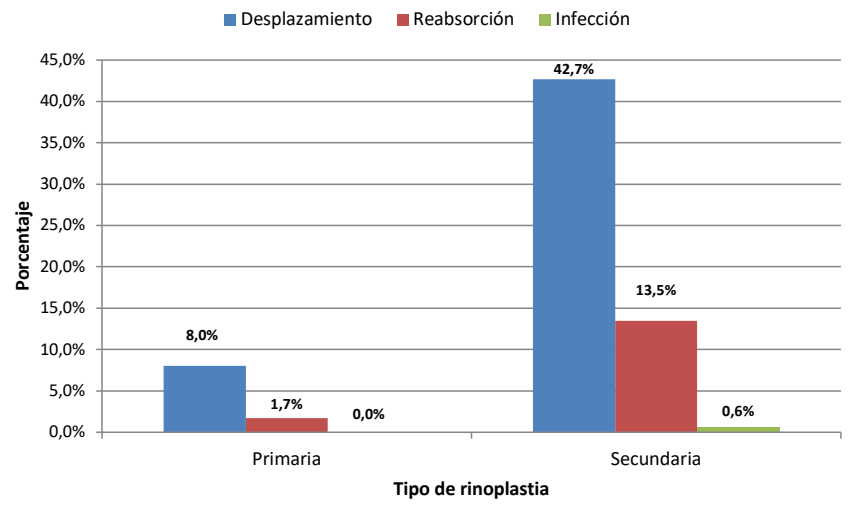

Figura 4. Distribución de las complicaciones estratificada por tipo de rinoplastia realizada. 


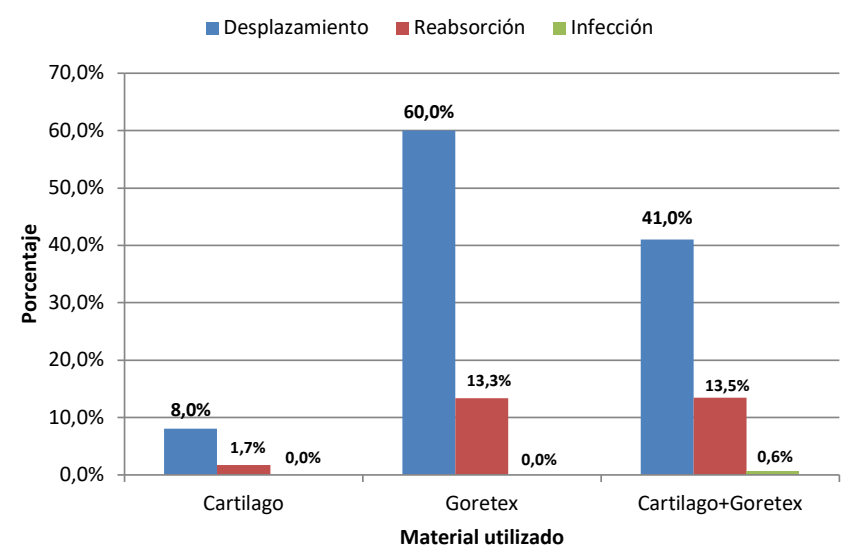

Gore-Tex®

Figura 5. Distribución de las complicaciones estratificada por el material del injerto CEP.

\section{Discusión}

En nuestro estudio el género femenino fue el grupo con el mayor porcentaje de rinoplastias, y con respecto a la estratificación por edad, los pacientes entre 20-30 años representaron la población mayoritaria. Lo anterior concuerda con la literatura científica en la que se evidencia que el género femenino se somete en mayor medida a cirugías plásticas, cuya razón psicológica se relaciona con el esquema activado de estándares inflexibles; esto significa que las mujeres tienen la necesidad de ser reconocidas por la sociedad y para ello adoptan los fenotipos de belleza establecidos por esta (15). [Termino correcto ]

Aunque nuestro estudio abarca a pacientes sometidos a una nueva técnica quirúrgica, al compararlo con otros trabajos en los que se utilizó el cartílago septal para la realización de la rinoplastia, encontramos tasas variables de complicaciones. Por ejemplo, en el trabajo ejecutado por Godfrey, en el que se utilizó cartílago septal morselizado, se reporta una tasa de complicaciones del $12,5 \%$, las cuales ocurrieron debido al desplazamiento del injerto y la ruptura, esta última relacionada con el uso de sutura no absorbible (16). En nuestro estudio no se presentó ruptura del injerto, pero el porcentaje de desplazamiento fue ligeramente superior $(14,5$ $\%)$. Sin embargo, cabe mencionar que, en el estudio de Godfrey, fue necesaria la utilización de mínimo dos piezas de cartílago en todos los pacientes debido a los antecedentes de procedimientos quirúrgicos en el cartílago septal, fragmentación o deformidad de este. Asimismo, en el $56 \%$ de los casos se requirió el uso de capas adicionales de cartílago debajo del injerto previamente realizado (16).

Por otro lado, nosotros solo utilizamos Gore-Tex ${ }^{\circledR}$ en $1,7 \%$ de los pacientes y una combinación de cartílago y Gore-Tex ${ }^{\circledR}$ en el $17,7 \%$. En estos dos grupos las complicaciones (desplazamiento y reabsorción) fueron mayores en comparación con el uso solamente de cartílago septal. Al comparar estos resultados con otros estudios reportados en la literatura, el estudio realizado por Goding y colabo- radores mostró que el 3,2 \% de los pacientes en los que se usó Gore-Tex ${ }^{\circledR}$ presentaron infección con la remoción del injerto, y hubo diferencias significativas en la tasa de infecciones de rinoplastias primarias en comparación con las rinoplastias de revisión (17). En nuestro estudio, el porcentaje de infección fue inferior al $1 \%$ y se presentó en un solo paciente sometido a rinoplastia secundaria, en el que se usaron materiales mixtos.

En contraposición a lo anterior, Lohuis y colaboradores en 2001 analizaron los resultados posquirúrgicos de 66 rinoplastias con corrección del dorso nasal con Gore-Tex®, en las que no se observaron complicaciones en las rinoplastias primarias ni secundarias (18). Por otro lado, Bracaglia y colaboradores siguieron 300 casos de rinoplastias de revisión en el transcurso de 1 año (147 injertos de cartílago, 89 injertos óseos, 10 faciales dérmicos o temporales y 42 Gore-Tex $\left.{ }^{\circledR}\right)$. En los 47 pacientes que recibieron GoreTex ${ }^{\circledR}$ por defectos menores del dorso nasal hubo una tasa de infección del $10 \%$ y 1 fístula cutánea desarrollada (19). Todos estos resultados fueron superiores a los descritos en el presente estudio.

Respecto al impacto del antecedente de cartílago traumatizado usado en rinoplastias, Mao y colaboradores desarrollaron un estudio en el que buscaban identificar ventajas y desventajas al utilizar cartílago para la corrección de la deformidad en silla de montar traumática. En este estudio realizaron un seguimiento a 20 pacientes con un tiempo promedio de 6,8 años, y se concluyó que el injerto de cartílago autógeno es útil para la corrección de este tipo de deformidades leves a moderadas gracias a los resultados positivos, funcionales y estéticos referidos por los pacientes durante el seguimiento; además, se encontraron complicaciones solo en un $5 \%$ ( 1 paciente) causadas por un edema en la punta de la nariz, pero no se encontraron reportes de infección, absorción ni desplazamiento del injerto (20). En nuestro trabajo no realizamos la distinción de pacientes con antecedentes de cartílago septal traumatizado, lo cual podría relacionarse con la complicación de desplazamiento reportada en nuestro grupo de pacientes.

La principal limitación del presente estudio radica en la ejecución de una técnica no descrita en la literatura, por ende, eso dificulta la comparación con otros abordajes quirúrgicos. Asimismo, el carácter retrospectivo limitó la consecución del tiempo exacto de aparición de la complicación y la identificación de las técnicas primarias realizadas en otras instituciones, de manera previa al abordaje con la técnica CEP. Finalmente, como aspecto positivo se destaca la disminución de las complicaciones a través de los años, lo que sugiere un perfeccionamiento de la técnica.

\section{Conclusiones}

La técnica CEP es un abordaje del dorso nasal no descrito previamente, que se realiza hace varios años en Cartagena de Indias. Las principales complicaciones con esta técnica fueron el desplazamiento y la reabsorción del injerto, las cuales 
predominaron en los pacientes en los que se utilizó el cartílago septal asociado a Gore-Tex ${ }^{\circledR}$ y en aquellas rinoplastias con el uso solamente de este material. Se necesitan más estudios para validar, perfeccionar y detectar posibles factores que influyan en la aparición de complicaciones

\section{Conflicto de interés}

Los autores no declaran ningún tipo de conflicto de interés

\section{Financiación}

No se requirió ningún tipo de financiación.

\section{Contribución de los autores}

Oñate-Carrillo contribuyó con la redacción de protocolo de investigación, la recolección de datos, la redacción de artículo, y la revisión y correcciones del documento. Montero-Cortés contribuyó con la recolección de datos, la redacción de artículo, y la revisión y correcciones del documento. Hortua-Bayona y Rojas-Salazar contribuyeron con la recolección de datos y la redacción de artículo.

\section{REFERENCIAS}

1. Niamtu J. Cosmetic Facial Surgery. 2.a edición. [San Luis]: Elsevier; 2018. 808 p.

2. Bagheri SC. Primary Cosmetic Rhinoplasty. Oral Maxillofac Surg Clin North Am. 2012;24(1):39-48. doi: 10.1016/j. coms.2011.10.001

3. Noemi LP. Cirugía estética de nariz. Rev Actual Clínica. 2014;47:2514-9.

4. Constantian MB. Rhinoplasty: Craft and Magic. 1.a edición. Nueva York: Thieme; 2009. 2302 p.

5. López-Cedrun JL. Cirugía reconstructiva y estética del tercio medio facial. 1.a edición. [Madrid]: Editorial ARAN; 2005. 366 p.

6. Fonseca R. Oral and Maxillofacial Surgery. 3.a edición. San Luis: Elsevier Saunders; 2017. 2696 p.

7. Adamson PA, Galli SK. Rhinoplasty approaches: current state of the art. Arch Facial Plast Surg. 2005;7(1):32-7. doi: 10.1001/ archfaci.7.1.32

8. Abarca AA, Naser GA, Pardo JJ. Aplicaciones de materiales aloplásticos en rinoplastía. Rev. Otorrinolaringol. Cir. Cabeza Cuello. 2010;70(3):259-64. doi: 10.4067/S071848162010000300010

9. Sajjadian A, Rubinstein R, Naghshineh N. Current status of grafts and implants in rhinoplasty: part I. Autologous grafts. Plast Reconstr Surg. 2010;125(2):40e-49e. doi: 10.1097/ PRS.0b013e3181c82f12

10. Sajjadian A, Naghshineh N, Rubinstein R. Current status of grafts and implants in rhinoplasty: Part II. Homologous grafts and allogenic implants. Plast Reconstr Surg. 2010;125(3):99e-109e. doi: 10.1097/PRS.0b013e3181cb662f

11. Escobar Sanz-Dranguet P, Márquez Dorsch F, Villacampa Aubá JM, Sanabria Brassart J, Gutiérrez Fonseca R, Pastormerlo Botegia $\mathrm{G}$, et al. Injertos cartilaginosos en rinoplastia. Acta Otorrinolaringol Esp. 2002;53(10):736-40.

12. Gunter JP RR. Augmentation rhinoplasty: dorsal onlay grafting using shaped autogenous septal cartilage. Plast Reconstr Surg. 1990;86(1):39-45.

13. Álvarez-Buylla Blanco M, Sarandeses García A, Chao Vieites J, Babarro Fernández R, Deus Abelenda C, Padín Seara A. Resultados funcionales y estéticos tras rinoplastia de aumento. Acta Otorrinolaringol Esp. 2011;62(5):347-54.

14. Lin G, Lawson W. Complications using grafts and implants in rhinoplasty. Oper Tech Otolaryngol - Head Neck Surg. 2007;18(4):315-23. doi: 10.1016/j.otot.2007.09.004

15. Moretti MP, Casari L. Satisfacción y motivación de la cirugía estética en mujeres y su relación con los esquemas desadaptativos tempranos. Psicogente. 2017;20(38):222-39. doi: $10.17081 /$ psico.20.38.2543

16. Godfrey NV. Augmentation rhinoplasty with mortised septal cartilage. Aesthetic Plast Surg. 1993;17(1):31-5. doi: 10.1007/ BF00455046

17. Godin MS, Waldman SR, Johnson CM Jr. Nasal augmentation using Gore-Tex. A 10-year experience. Arch Facial Plast Surg. 1999;1(2):118-21; discussion 122. doi: 10.1001/ archfaci.1.2.118

18. Lohuis PJ, Watts SJ, Vuyk HD. Augmentation of the nasal dorsum using Gore-Tex: intermediate results of a retrospective analysis of experience in 66 patients. Clin Otolaryngol Allied Sci. 2001;26(3):214-7. doi: 10.1046/j.1365-2273.2001.00453.x

19. Bracaglia R, Fortunato R, Gentileschi S. Secondary rhinoplasty. Aesthetic Plast Surg. 2005;29(4):230-9. doi: 10.1007/s00266005-0034-Z

20. Mao J, Carron M, Tomovic S, Narasimhan K, Allen S, Mathog RH. Cartilage grafts in dorsal nasal augmentation of traumatic saddle nose deformity: a long-term follow-up. Laryngoscope. 2009;119(11):2111-7. doi: 10.1002/lary.20491 